\section{Commentary: Recovering ischemic myocardium - hibernation, autophagy, preconditioning, mitochondria, stem cells, and more}

Victor A. Ferraris, MD, PhD

Approaches to improve cardiac function following ischemic stress are multiple and account for a significant contribution to the current literature. In this issue of the Journal, an article by Hocum-Stone and colleagues ${ }^{1}$ describes a novel means of moderating ischemic cardiac stress. These authors developed an animal model of intraoperative placement of mesenchymal stem cell patches to improve myocardial function in an animal model of hibernating myocardium. The authors investigated some of the intermediate metabolism in mitochondria that leads to hibernation and that may be responsible for ischemic preconditioning and ultimately recovery of cardiac cellular function. These authors were able to develop an animal model of hibernating myocardium using a subacute coronary artery narrowing to create an ischemic focus in a large animal porcine model. They subsequently performed a unique reperfusion intervention at the time of coronary revascularization using a synthetic patch impregnated with mesenchymal stem cells overlying an ischemic focus on the myocardium.

Ischemic cardiac cells rely on mitochondria for cell survival, both for ATP synthesis and for $\mathrm{Ca}^{2+}$ homeostasis. Mitochondrial membranes and DNA are damaged by ischemic injury. One of the most intriguing observations about myocardial ischemic injury is that during ischemia, an alteration in intracellular $\mathrm{Ca}^{2+}$ homeostasis occurs and mitochondria are able to buffer cytosolic $\mathrm{Ca}^{2+}$ and retain the calcium-transporting capacity up to a point of

\footnotetext{
From the Department of Surgery, University of Kentucky, Lexington, Ky.

Disclosures: Author has nothing to disclose with regard to commercial support.

Received for publication Jan 1, 2020; accepted for publication Jan 2, 2020; available ahead of print Jan 11, 2020.

Address for reprints: Victor A. Ferraris, MD, PhD, Department of Surgery, University of Kentucky, A301 Kentucky Clinic, 740 South Limestone, Lexington, KY 405360284 (E-mail: ferraris@uky.edu).

J Thorac Cardiovasc Surg 2021;162:e17-8

0022-5223/ $\$ 36.00$

Copyright () 2020 by The American Association for Thoracic Surgery

https://doi.org/10.1016/j.jtcvs.2020.01.002
}

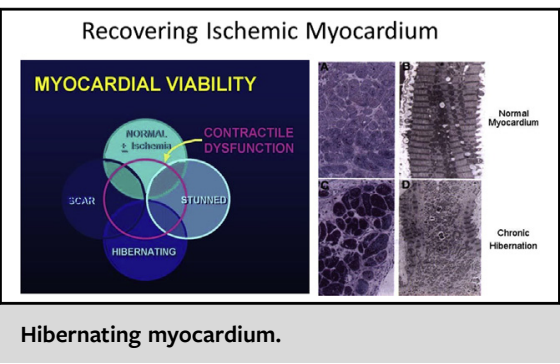

CENTRAL MESSAGE

There are multiple poorly understood mechanisms that can help support damaged myocardium. Mesenchymal stem cells may prove to be useful in helping damaged myocardium recover.

irreversibility. ${ }^{2}$ Accordingly, even after prolonged ischemia, the majority of the cellular mitochondria are able to use oxygen for ATP phosphorylation and subsequent energy generation, although not nearly at normal levels. When isolated after reperfusion, mitochondria are structurally altered, contain large quantities of $\mathrm{Ca}^{2+}$, and produce an excess of oxygen-free radicals. Damaged mitochondria undergo dramatic changes including fusion, fragmentation, and hibernation.

At some point after prolonged ischemic stress, mitochondrial membranes are disrupted and the important function of oxidative phosphorylation ceases, but there is a critical time during which interventions and reperfusion can salvage myocardial mitochondrial viability. These salvaged mitochondria are not irreversibly damaged and are part of the picture of hibernating myocardium. The good part about hibernating myocardium is that it is not "dead." The "bad" part is that it is not fully functional and continues to have decreased function despite apparent adequate oxygen deliveryhence the term hibernation.

As the authors of this manuscript point out, postischemia recovery of mitochondrial function is a complex and multifaceted process that involves fusion, fission, and hibernation of mitochondria. These findings are consistent with preclinical studies indicating that homeostatic intracellular repair response is cardioprotective and that it is activated in patients in response to myocardial ischemic stress. ${ }^{3}$ Autophagy, the process of recycling organelles and protein aggregates, is a basic homeostatic 
process and an evolutionarily conserved response to starvation and other forms of metabolic stress. ${ }^{3}$ Likewise, recovery of heart mitochondrial function in response to ischemic stress appears to be an important response to ischemic stress. This mitochondrial response is dependent on a dynamic balance of several processes, including disruption of nonfunctioning mitochondria with salvage of mitochondrial contents in the cytoplasm (a process labeled as "fission" by the authors but containing many of the elements of autophagy), and a process of "fusion," where marginally functioning mitochondria can combine resources by fusing together. ${ }^{4,5}$ These processes are aimed at helping ischemic cells manage ischemic stress by conserving important cell-sustaining mechanisms especially those dependent on mitochondria. As the authors point out, these processes are not perfect. Fusion of damaged mitochondria results in somewhat-imperfect mitochondria that are small and not as competent as a normal mitochondrion but are still alive and ultimately able to function by providing cell viability.

Current in vitro cellular studies showed that ischemic conditioning improves the contractile recovery of isolated ventricular papillary muscle or atrial trabeculae following simulated ischemia. However, in vivo animal studies and studies in patients undergoing elective cardiac surgery show conflicting results, with a much less dramatic enhancement of cardiac function in myocardium subjected to ischemic conditioning. ${ }^{6}$ Prolonged cardiac ischemia time appears to worsen subsarcolemmal mitochondrial function and $\mathrm{Ca}^{2+}$ homeostasis. ${ }^{7}$ In contrast, intermittent hypoxia seems to maintain $\mathrm{Ca}^{2+}$ homeostasis and mitochondrial membrane integrity during oxidative stress inducedischemia, although at a cost of cell function. ${ }^{8}$ These recent experimental observations are neither novel nor new. Many publications attest to the value of ischemic preconditioning in maintenance of viable mitochondria using in vitro experiments. There have been far fewer studies that show in vivo augmentation of myocardial energetics from hibernating myocardium after successful revascularization. In this regard, the manuscript by Hocum Stone and colleagues is novel and provides some important and intriguing evidence that stem cell-related interactions with ischemic myocardium can provide meaningful help in ischemic stress recovery.

So where do stem cells come into the picture? The sad truth is that there is no uniformly agreed-on mechanism of benefit from mesenchymal stem cells. Most of what we know about stem cell benefits is what stem cells don't do. There is very limited evidence that stem cells can differentiate into viable functioning myocardial cells. The recent postulated benefit of stem cells is a mysterious paracrine mechanism without much real evidence to support this thought. As the authors suggest, mesenchymal stem cell treatment in animal studies is associated with increased expression of nuclear factors, one of which is PGC1alpha. Exactly how stem cells can cause this expression of nuclear factors is uncertain but remains an area of ongoing research. There is some very provocative work that suggests that the benefit from mesenchymal stem cells is related to mitochondrial function. The current manuscript supports this notion indirectly, but much more work needs to be done, and there is an important and pressing need for these results.

\section{References}

1. Stone LLH, Swingen C, Wright C, Qi SS, Rassette M, McFalls EO, et al. Recovery of hibernating myocardium using stem cell patch with coronary bypass surgery. J Thorac Cardiovasc Surg. 2021;161:e3-16.

2. Ferrari R. The role of mitochondria in ischemic heart disease. J Cardiovasc Pharmacol. 1996;28(suppl 1):S1-10.

3. Jahania SM, Sengstock D, Vaitkevicius P, Andres A, Ito BR, Gottlieb RA, et al. Activation of the homeostatic intracellular repair response during cardiac surgery. J Am Coll Surg. 2013;216:719-26; discussion 726-729.

4. Chen H, Chan DC. Physiological functions of mitochondrial fusion. Ann N Y Acad Sci. 2010;1201:21-5.

5. Gustafsson AB, Gottlieb RA. Autophagy in ischemic heart disease. Circ Res. 2009; 104:150-8.

6. Stoian L, Kruger M, Schmitt J, Kleinbongard P. Is there an effect of ischemic conditioning on myocardial contractile function following acute myocardial ischemia/reperfusion injury? Biochim Biophys Acta Mol Basis Dis. 2019;1865: 822-30.

7. Leistner M, Sommer S, Kanofsky P, Leyh R, Sommer SP. Ischemia time impacts on respiratory chain functions and $\mathrm{Ca}(2+)$-handling of cardiac subsarcolemmal mitochondria subjected to ischemia reperfusion injury. J Cardiothorac Surg. 2019; $14: 92$

8. Chang JC, Lien CF, Lee WS, Chang HR, Hsu YC, Luo YP, et al. Intermittent hypoxia prevents myocardial mitochondrial $\mathrm{Ca}(2+)$ overload and cell death during ischemia/reperfusion: the role of reactive oxygen species. Cells. $2019 ; 8$. 